Military Technical College Kobry El-Kobbah, Cairo, Egypt

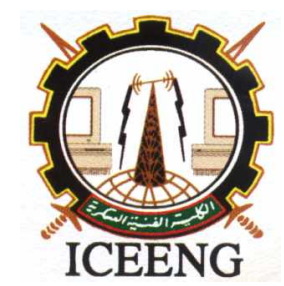

\author{
$6^{\text {th }}$ International Conference \\ on Electrical Engineering \\ ICEENG 2008
}

\title{
Multisensor Data Fusion Fuzzy Similarity-based of Several Kalman Filters
}

\author{
By
}

Ahmed E. Abdalla*

\section{Abstract:}

The main objective of this work is the development of an intelligent multisensor integration and fusion model that uses fuzzy similarity-based data fusion of several Kalman filters outputs. First, the estimation of sensors outputs are calculated using a set of Kalman filters with pre-estimated measurement noise. Using fuzzy set theory, the fuzzy similarity between the predicted data is extracted to determine the importance weight of each sensor. Weights assigned to different sensors measurement data to reflect the confidence in the sensor's behavior and performance and to realize the multi-sensor data fusion. According to the algorithm theory, its application software is developed using MATLAB. This work has wide applications especially in the development of radar target tracking, smart structural health monitoring systems, biomedical imaging, and robotics control. The applied example proves that the algorithm can give priority to the high-reliability and stability sensors. Moreover, it reflects the efficiency and feasibility to real-time data processing and monitoring.

\section{Keywords:}

Kalman Fiter, Fuzzy similarity measurement, and multisensor data fusion. 


\section{Introduction:}

In recent years, multisensor data fusion has received a special attention for a wide of applications which require accurate measurements of measured parameters [1-4]. Accuracy in measuring the state of a dynamic stochastic system is difficult using a single sensor. In many cases process and measurements noise cannot be ignored. Therefore, a reliable procedure is needed to estimate the measured signal correctly. A Kalman filter is used extensively for signal estimation. The Kalman filter is an optimal linear estimator that provides the estimation of the signal in noise [4] and is known as a powerful algorithm for estimation. It has the features of unbiased with linear estimate and produces estimate with minimum error covariance. However, the Kalman filter is based on known characteristics of the system. These characteristics are difficult to determine in many real life applications [5].

Extending the Kalman filter using suitably defined estimation algorithms has shown improved results [6]. One way to improve accuracy is by combining multiple sensors measuring the same parameter into one logical component With this approach, the effect of problems associated with sensors may be minimized. Multi-Sensor data fusion is advantageous because the strengths of one sensor often compensate for the weaknesses of the other. Earlier work [7] developed certain rules by which data from sensors were taken. Data fusion of different sensors based on Kalman filter has been reported by many authors to control movement of wheeled mobile robot [8]. After successful estimation, or prediction, the data of the sensors are fused together to form one logical sensor $[9,10]$. Different methods for connecting the Kalman filter to sensors were used [11].

This paper presents architecture for fusing data obtained from several sensors. The presented architecture is based on adaptive Kalman filter connected to each sensor. Output of these filters was fused together to perform predicted output. The general idea explored in this approach is the combination of the advantages that both Kalman filtering and fuzzy logic similarity techniques [12]. On the one hand, Kalman filtering is acknowledged as one of the most powerful traditional techniques of estimation. On the other hand, the main advantages derived from the use of fuzzy logic similarity techniques, with respect to traditional schemes, are the simplicity of the approach, the capability of fuzzy systems to deal with imprecise information, and the possibility of including heuristic knowledge about the phenomenon under consideration required. In addition to the capability of combining information from different sensors, the proposed architecture overcomes on the degradation of the overall performance if some sensors units fail or interconnections are broken. 


\section{Kalman Filter}

\subsection{Basics principle of Kalman Filter}

The Kalman filter provides an efficient computational( recursive) means to estimate the state of a process, in a way that minimizes the mean of the squared error. The filter is very powerful in several facets: it supports estimations of past, present, and even future states, and it can be done so even when the accurate nature of the modeled system is unknown.

The Kalman filter addresses the general problem of trying to estimate the state of a $x \in \mathfrak{R}^{n}$ discrete-time controlled process that is governed by the linear stochastic difference equation

$$
x_{k}=A x_{k-1}+B u_{k-1}+w_{k-1} \text {, }
$$

with a measurement $z \in \mathfrak{R}^{n}$ that is

$$
z_{k}=H x_{k-1}+v_{k-1}
$$

The random variables $\omega$ and $v$ represent the process and measurement noise (respectively). They are assumed to be independent (of each other), white, and with normal probability distributions

$$
\begin{aligned}
& P(\omega) \sim N(0, Q) \\
& P(\mathrm{v}) \sim N(0, R)
\end{aligned}
$$

The matrix $n x n$ in the difference equation (1) relates the state at the previous time step $\mathrm{k}-1$ to the state at the current step $\mathrm{k}$, in the absence of either a driving function or process noise. Note that in practice might change with each time step, but here we assume it is constant. The $n x l$ matrix $\mathrm{B}$ relates the optional control input $u \in \mathfrak{R}^{l}$ to the state $\mathrm{x}$. The $m x n$ matrix $\mathrm{H}$ in the measurement equation (2) relates the state to the measurement $z_{k}$. In practice $\mathrm{H}$ might change with each time step or measurement, but here we assume it is constant.

We define $\dot{x}_{k}^{-} \in \mathfrak{R}^{n} \quad$ to be our a priori state estimate at step $k$ given knowledge of the process prior to step $k$ and $x_{k} \in \mathfrak{R}^{n}$ to be our a posteriori state estimate e at step $k$ given measurement $z_{k}$. We can then define a priori and a posteriori estimate errors as $e_{k}^{-} \equiv x_{k}-\hat{x}_{k}^{-}$and $e_{k} \equiv x_{k}-\hat{x}_{k}$

The a priori estimate error covariance is then

$$
P_{k}^{-}=E\left[e_{k}^{-}-e_{k}^{-T}\right]
$$

and the a posteriori estimate error covariance is

$$
P_{k}=E\left[e_{k}-e_{k}^{T}\right\rfloor
$$

The Probabilistic origins of the Kalman filter found below. 
$\hat{x}_{k}=\hat{x}_{k}^{-}+K\left(z_{k}-H \hat{x}_{k}^{-}\right)$

The difference $\left(z_{k}-H \hat{x}_{k}^{-}\right)$in (7) is called the measurement innovation, or the residual. The residual reflects the discrepancy between the predicted measurement $H \hat{x}_{k}^{-}$and the actual measurement $z_{k}$ A residual of zero means that the two are in complete agreement.

The difference $\left(z_{k}-H \hat{x}_{k}^{-}\right)$in (7) is called the measurement innovation, or the residual. The residual reflects the difference between the predicted measurement $H \hat{x}_{k}^{-}$, and the actual measurement zk. A residual of zero means that the two are in complete agreement The nxm matrix $\mathrm{K}$ in (7) is chosen to be the gain or blending factor that minimizes the a posteriori error covariance (6). This minimization can be accomplished by first substituting (7) into the above definition for $\mathrm{e}_{\mathrm{k}}$, substituting that into (6). Performing the indicated expectations, taking the derivative of the trace of the result with respect to K. setting that result equal to zero, and then solving for K. For more details see [5]. One form of the resulting $\mathrm{A}^{\prime}$ that minimizes (6) is given by

$$
K_{k}=P_{k}^{-} H^{T}\left(H P_{k}^{-} H^{T}+R\right)^{-1}
$$

\subsection{The Discrete Kalman Filter Algorithm}

The Kalman filter estimates a process by using a form of feedback control: the filter estimates the process state at some time and then obtains feedback in the form of (noisy) measurements. As such, the equations for the Kalman filter fall into two sets: time update equations and measurement update equations. The time update equations are responsible for projecting forward the current state and error covariance estimates to obtain the a priori estimates for the next time step. The measurement update equations are responsible for the feedback i.e. for incorporating a new measurement into the a priori estimate to obtain an improved a posteriori estimate. The time update equations can also be thought of as predictor equations, while the measurement update equations can be thought of as corrector equations. The final estimation algorithm look likes that of a predictor-corrector algorithm for solving numerical problems [5] as shown below in Fig. (1). 


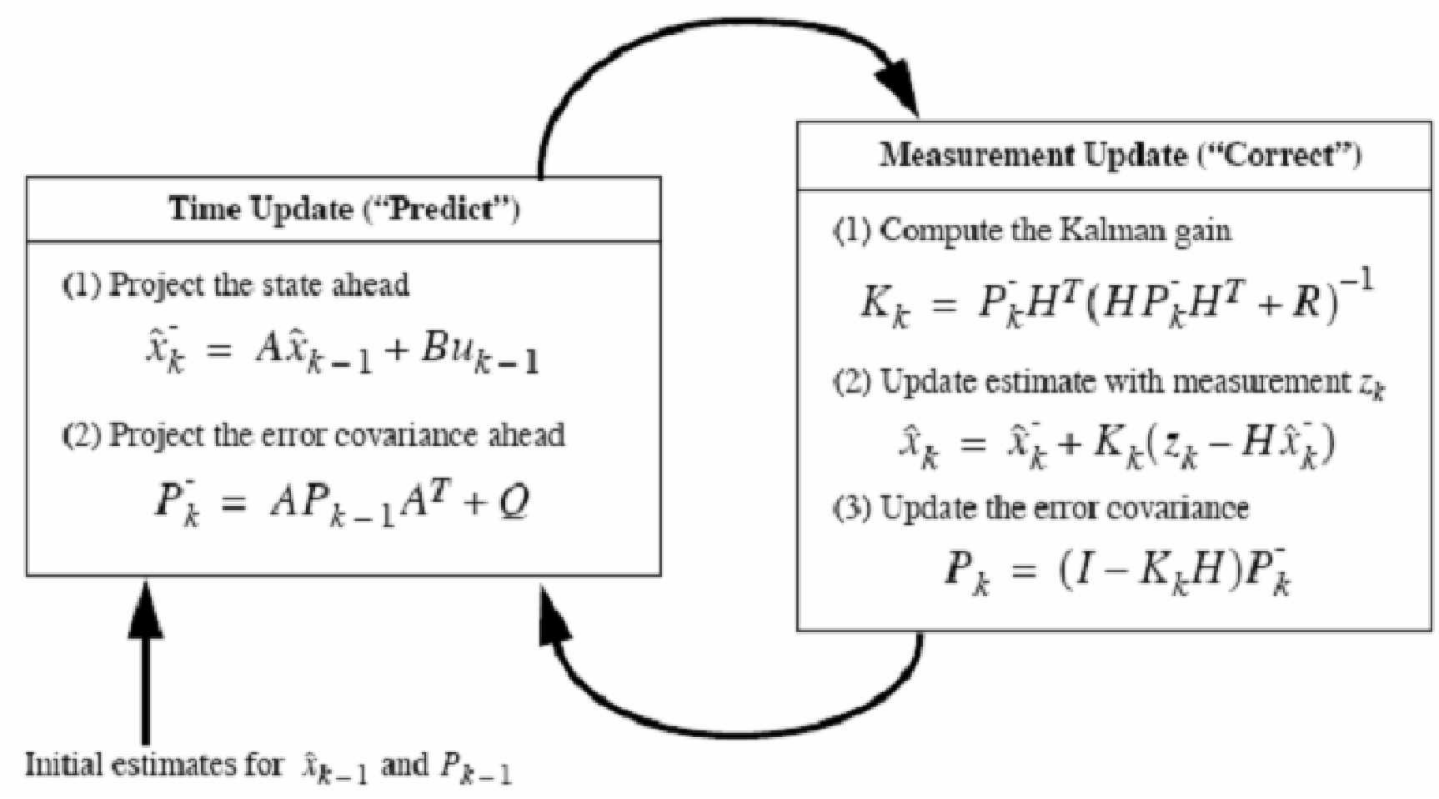

Fig. (1) A complete picture of the operation of the Kalman filter.

\section{$\underline{\text { 2.3 Filter Parameters and Tuning }}$}

In the actual implementation of the filter, the measurement noise covariance $\mathrm{R}$ is usually measured prior to operation of the filter. Measuring the measurement error covariance $\mathrm{R}$ is generally practical possible because we need to be able to measure the process anyway while operating the filter so we should generally be able to take some off-line sample measurements in order to determine the variance of the measurement noise.

The determination of the process noise covariance $\mathrm{Q}$ is generally more difficult as we typically do not have the ability to directly observe the process we are estimating. Sometimes a relatively simple process model can produce acceptable results if one considers uncertainty into the process by the selection of $\mathrm{Q}$. The tuning is usually performed off-line, frequently with the help of another Kalman filter in a process generally referred to as system identification.

\section{Fuzzy Similarity-based Data Fusion Algorithm}

We assume that there is a multi-sensor system with $\mathrm{n}$ sensors used to carry through measurement. A sample data $z_{i}$ from each i sensor is captured to pre-estimate offline the covariance matrix $R_{i}$ of measurement noise $v_{i}$ and $i=1,2, \ldots, n$. An $m$ Predicted values $x_{i j}$ of sensor $\mathrm{i}$ is extracted simultaneously using $\mathrm{m}$ Kalman filters with different covariance matrix $R_{i}+\Delta R_{i j}(j=1,2, \ldots m)$ and thereby gets an $m$ estimated measurement values: $\mathrm{x}_{\mathrm{i} 1}, \mathrm{x}_{\mathrm{i} 2}, \ldots, \mathrm{x}_{\mathrm{im}}$. $(\mathrm{i}=1,2,3,---, \mathrm{n})$. Then the fuzzy similarity-based multi-sensor data 
fusion algorithm is used in four stages.

First stage, data from multiple sensors is extracted. The value $x_{i}$ mean measurement $i$, standard deviation $\sigma_{j}$, of the i's sensor. The multiple sensors objective prediction value $\mathrm{x}_{0}$ and standard deviation $\sigma_{0}$ are calculated.

$$
\begin{aligned}
& x_{i}=\frac{1}{k} \sum_{j=1}^{m} x_{i j}, \sigma_{i}=\sqrt{\frac{1}{m-1}} \sum_{j=1}^{m}\left(x_{i j}-x_{i}\right)^{2}, \mathrm{i}=1,2, \ldots, \mathrm{n} . \\
& x_{0}=\frac{1}{k} \sum_{i=1}^{n} x_{i}, \sigma_{0}=\sqrt{\frac{1}{n-1} n \sum_{j=1}^{m}\left(x_{i}-x_{0}\right)^{2},}
\end{aligned}
$$

Second stage, Grubbs judgment algorithm is used to eliminate those unrealistic data. We arrange $\mathrm{x}_{1} \mathrm{x}_{2}, \mathrm{x}_{3}, \ldots, \mathrm{x}_{\mathrm{n}}$ from minimum to maximum and give the significance level $\alpha$ [13-14]. Assuming that Grubbs statistic $h_{i}=\frac{x_{i}-x_{0}}{\sigma_{0}}$. If this Grubbs statistic $h_{i}$ is an outlier, then the wrongly measured $\mathrm{x}_{\mathrm{i}}$ is eliminated.

Third stage, according to fuzzy set theory, a certain sensor's measurement values and the multiple sensors' prediction values are respectively expressed as fuzzy sets $F_{i}$ and $F_{0}$. The fuzzy similarity between them is used to determine the importance weigh of a certain sensor. Let normal fuzzy sets $\mathrm{F}_{\mathrm{i}}$ and $\mathrm{F}_{\mathbf{0}}$ denote respectively as the fuzzy sets of the i's sensor measurement values $x_{i}$ and the multiple sensors objective prediction values $\mathrm{x}_{0}$.

$\mu_{F_{i}}(t)=\exp \left[-\left(\frac{t-x_{i}}{\sigma_{i}}\right)^{2}\right]$
$\mu_{F 0}(t)=\exp \left[-\left(\frac{t-x_{i}}{\sigma_{n}}\right)^{2}\right]$

We can know that the similarity between fuzzy sets $F_{i}$ and $\mathbf{F}_{\mathbf{0}}$

$\eta\left(F_{i}, F_{0}\right)=\exp \left[-\left(\frac{x_{i}-x_{0}}{\sigma_{i}+\sigma_{0}}\right)^{2}\right]$

$\eta\left(F_{i}, F_{0}\right)$ is higher, $\mathrm{F}_{\mathrm{i}}$ is closer to $\mathrm{F}_{0}, \eta\left(F_{i}, F_{0}\right)$ can be viewed as the importance weigh of the i sensor during the data fusion. 
Last stage, we calculate each sensor's relative importance weigh

$$
\omega_{i}=\frac{\eta\left(F_{i}, F_{0}\right)}{\sum_{i=1}^{n} \eta\left(F_{i}, F_{0}\right)},(\mathrm{i}=1,2, \ldots, \mathrm{n})
$$

The final data fusion is calculated as:

$$
z=\sum_{i=1}^{n} w_{i} x_{i}
$$

Fig. (2) shows the sequence steps of proposed multi sensor data fusion architecture.

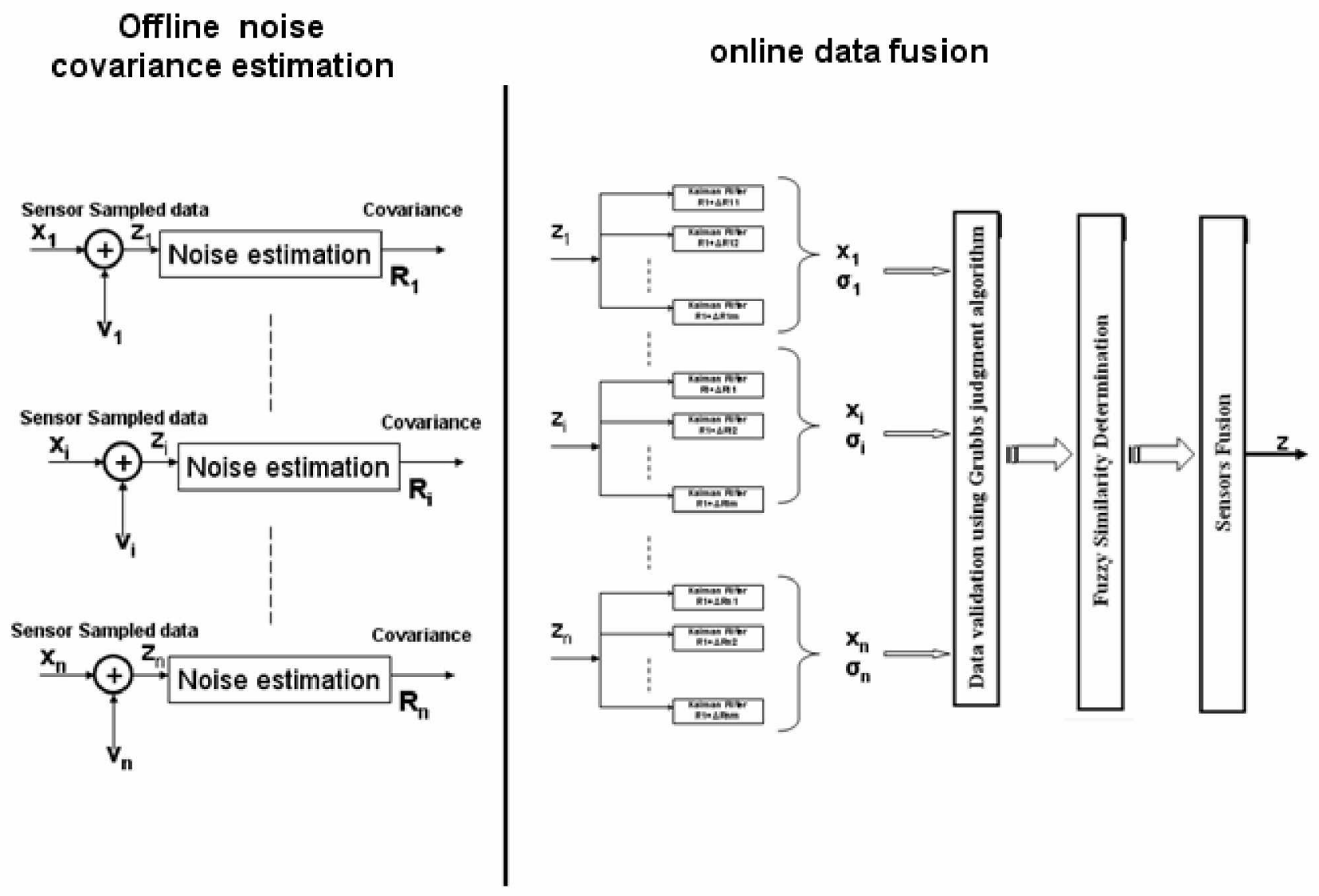

Fig. (2) Proposed multisensor data fusion architecture 


\section{Illustrative Example}

To demonstrate the effectiveness and accuracy of this proposed method of multisensor Data Fusion architecture, an example with three noisy sensors is outlined. Consider the following linear system,

$$
\begin{aligned}
& x_{k+1}=\left[\begin{array}{ccc}
1.1269 & -0.4940 & 0.1129 \\
1.0 & 0.0 & 0.0 \\
0.0 & 1.0 & 0
\end{array}\right] x_{k}+\left[\begin{array}{c}
-0.3832 \\
0.5919 \\
0.5919
\end{array}\right] u_{k}+w_{k} \\
& z_{k}=\left[\begin{array}{lll}
1 & 0 & 0
\end{array}\right] x_{k}+v_{k}
\end{aligned}
$$

With initial conditions $x_{0}=0$, in the equation (16) the system noise sequence $w_{k}$ is a zero-mean Gaussian white noise sequence with covariance $Q=1$. The measurement noise sequence $v_{k}$ is generated as zero-mean Gaussian white noise sequence with covariance $R_{i}$ starting value pre-estimated from a measurement sample data for each senor $i$. The change vector of covariance $R_{i}$ is taken as

$$
\Delta R=\left[\begin{array}{lllll}
0 & -0.1 & 0.1 & -0.2 & 0.2
\end{array}\right] \text {. }
$$

A Matlab code was developed to simulate the process described by equation (16). The actual value of $\mathrm{R}$ for each sensor is assumed unknown starting value which is estimated from a sampled measurement data offline as shown in Fig. (3).

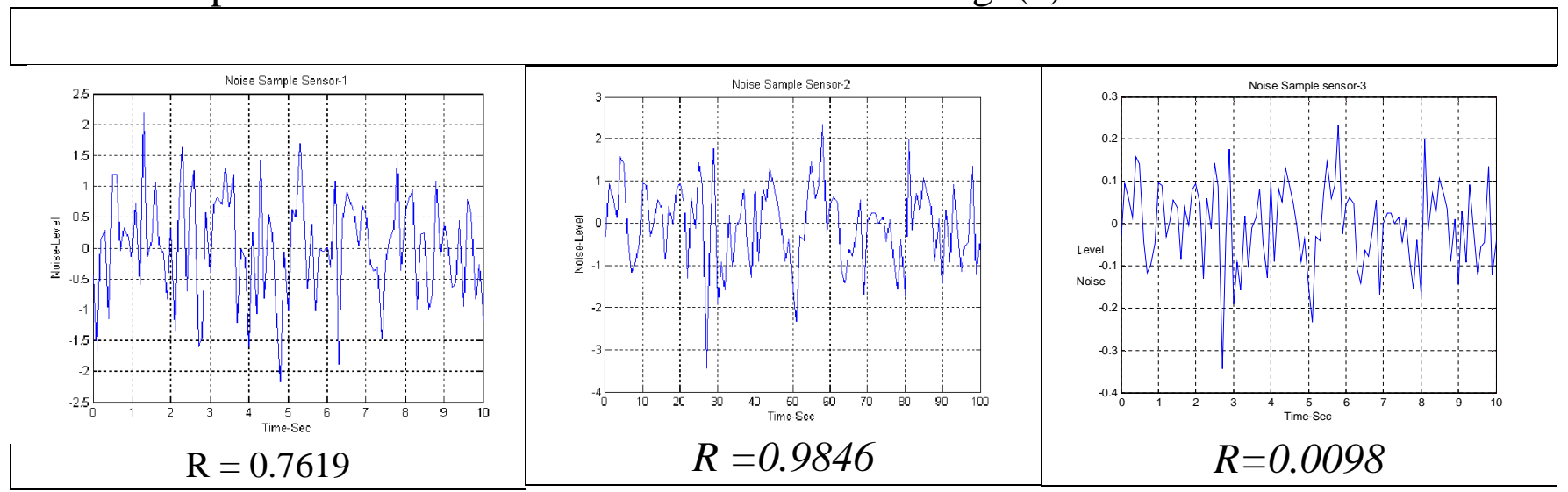

Figure (3): Sampled data noise for covariance estimation of each sensor 


$$
\text { output } \begin{aligned}
& --_{\text {true }} \\
& - \text { estimated }
\end{aligned} \text { error } \begin{aligned}
& --\cdot \text { true } \\
& \text { estimated }
\end{aligned}
$$
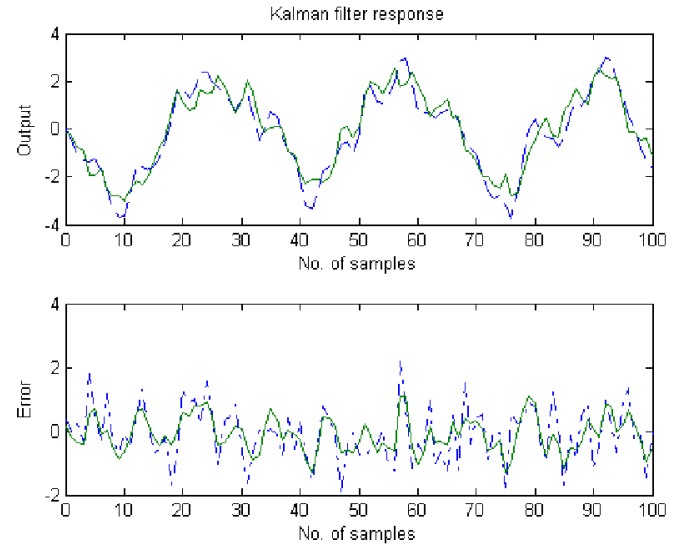

(a)
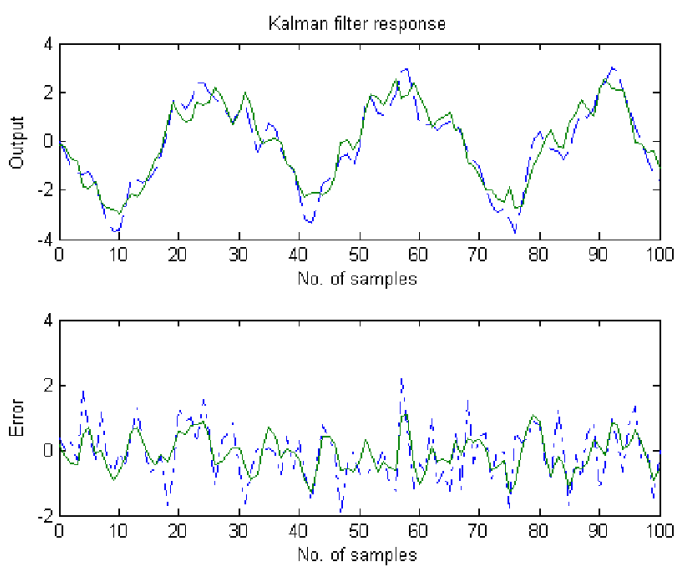

(c)
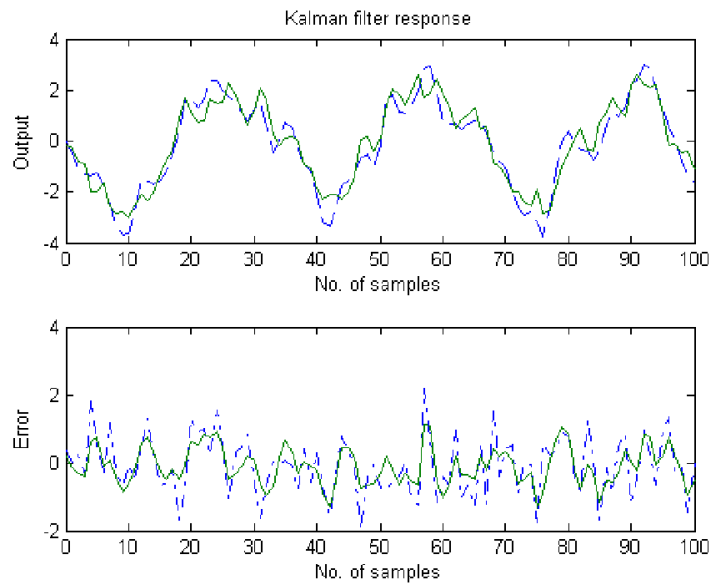

(b)
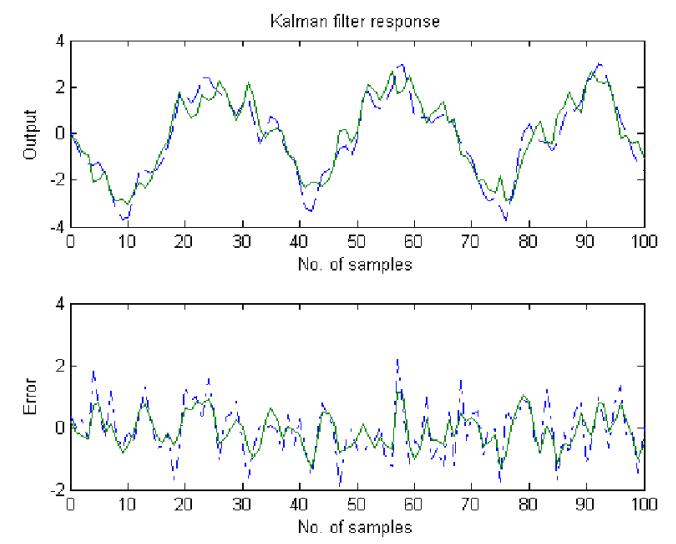

(d)
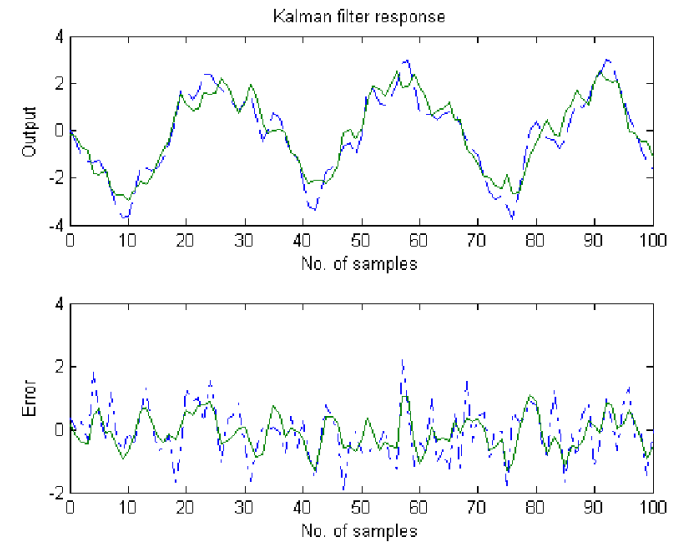

(e)

Fig. (4): Kalman filter response of sensorl with different covariance matrix and measurement error 


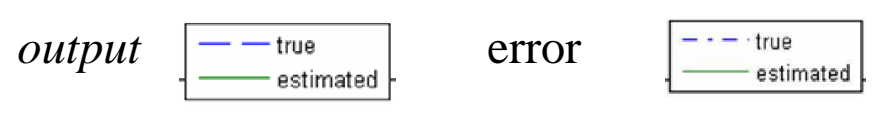
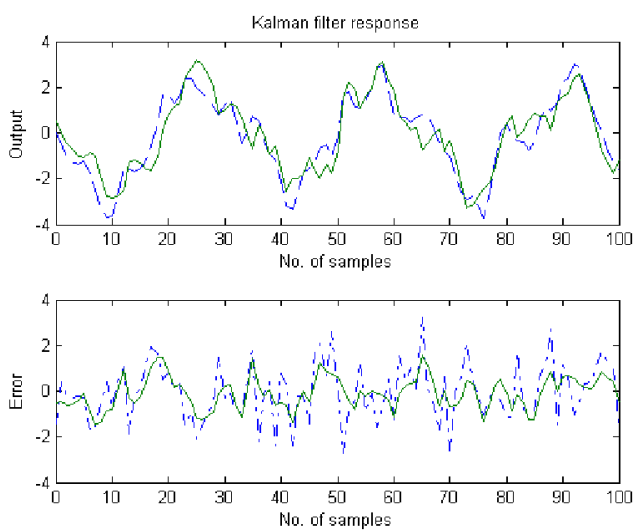

(a)
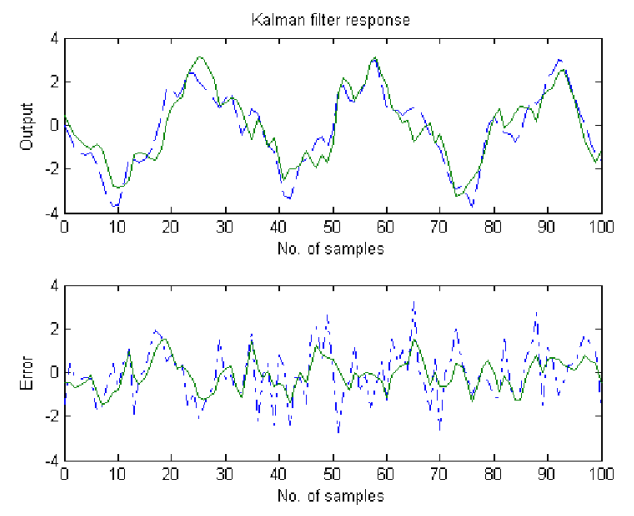

(c)
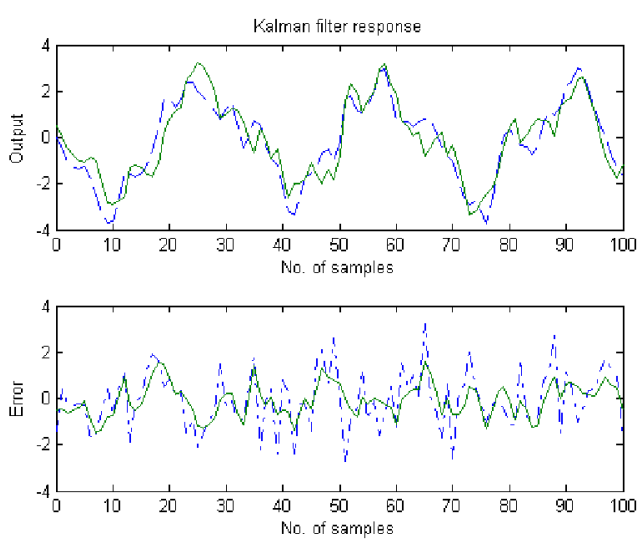

(b)
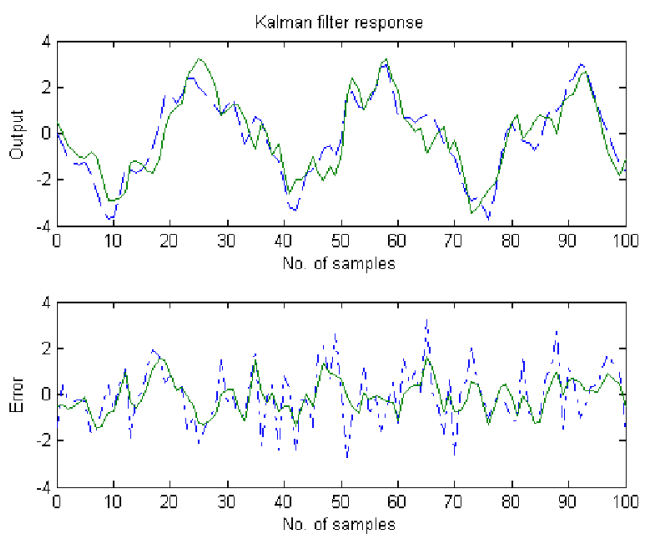

(d)
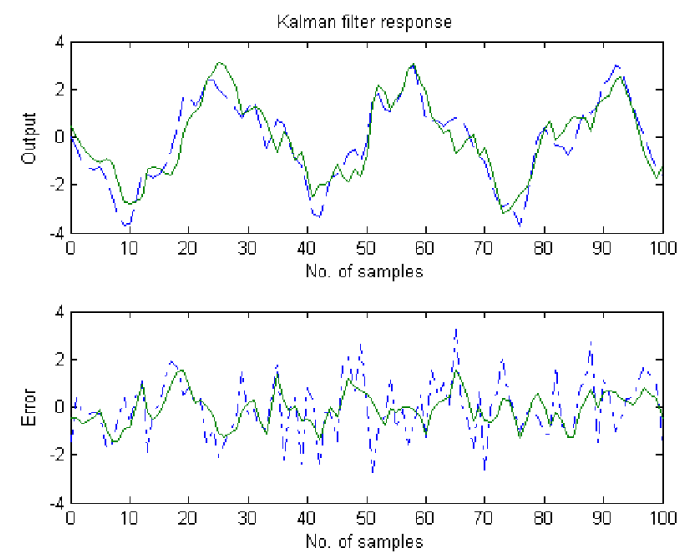

(e)

Fig. (5):Kalman filter response of sensor 2 with different covariance matrix and measurement error 


\section{output $\quad$ error}
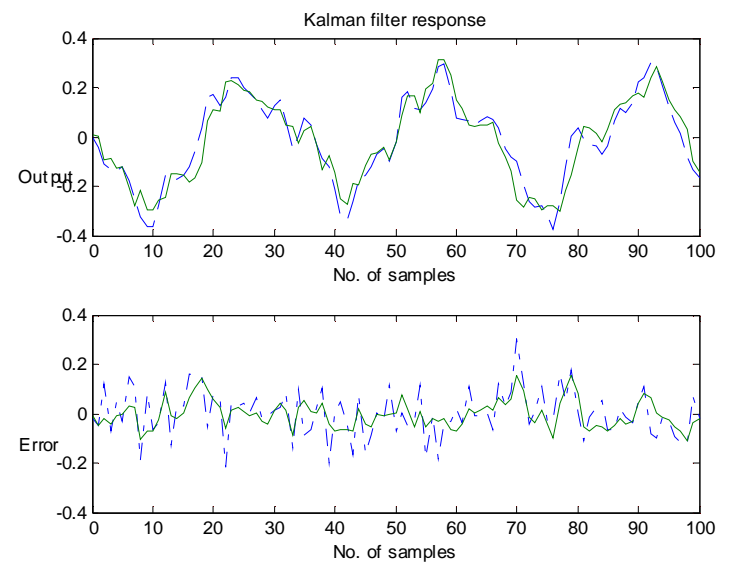

(a)
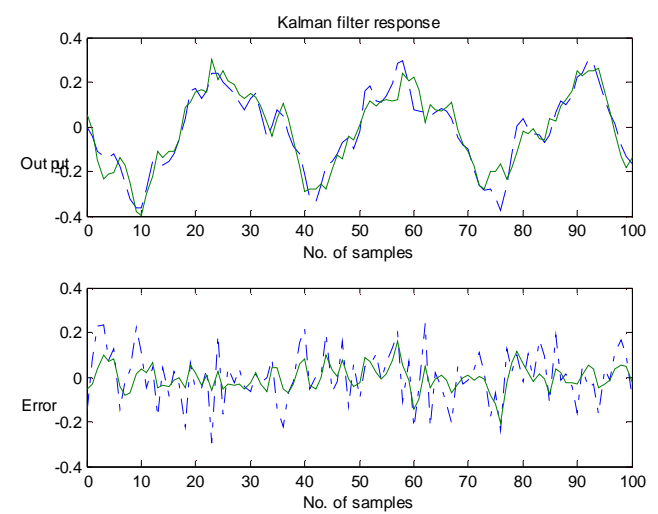

(c)
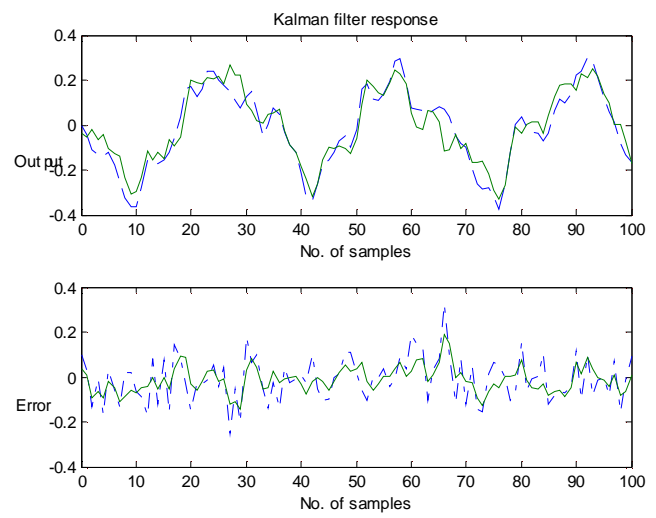

(b)
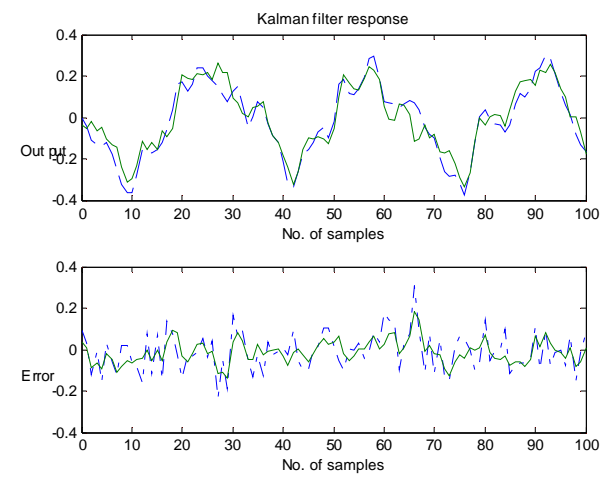

(d)
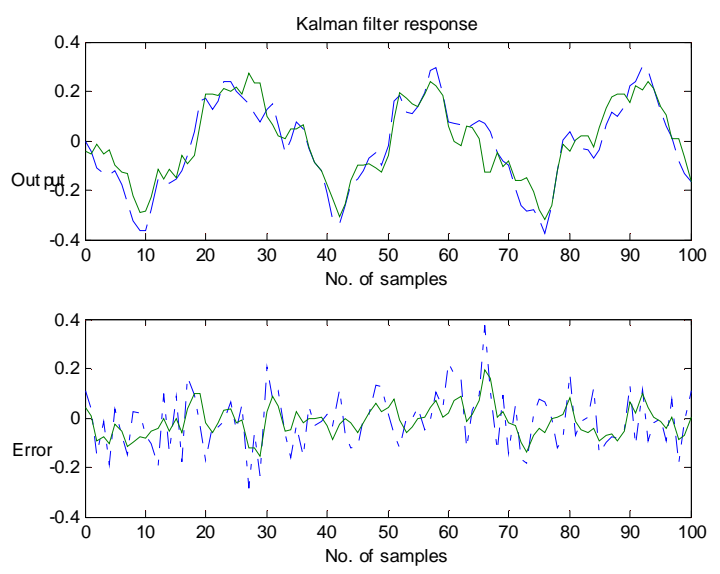

(e)

Fig. (6): Kalman filter response of sensor3 with different covariance matrix and measurement error 


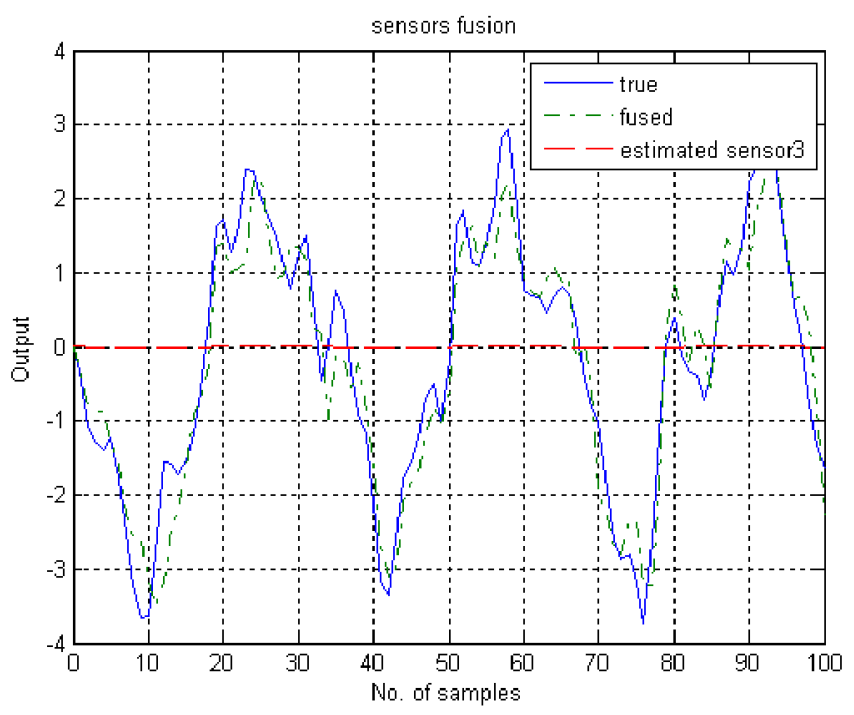

Figure (7): Fused response and Kalman filter response of failed sensor3

Table (1): Statistical Error Analysis

\begin{tabular}{|c|c|c|}
\hline sensor & Mean & Standard Deviation \\
\hline Sensor1 & 0.0550 & 0.5106 \\
\hline Sensor2 & 0.0596 & 0.5310 \\
\hline Sensor3 & 0.0014 & 0.0079 \\
\hline Fused & 0.0660 & 0.5088 \\
\hline
\end{tabular}

Kalman filter response of sensor, sensor2, and sensors 3 using different measurement noise covariance $\mathrm{R}_{\mathrm{i}}$ changed around the estimated values from calculated one off-line

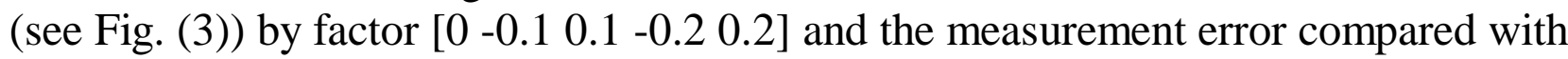
the true one are shown in Fig. (4), Fig. (5), and Fig. (6) respectively. As it is clear from Fig. (6) we assume failure of data measurement of sensor 3. In spite of this failure, the system able to compensate it and fused only the realistic data as shown in Fig. (7). Statistical parameters of error measurements for each sensor compared with the fused one are given in table 1.

\section{Conclusions}

A multisensor data fusion architecture using a group of a set of Kalman filters and fuzzy similarity based techniques has been presented. This approach exploits the advantages that both approaches have: the optimality of the Kalman filter and the capability of fuzzy similarity systems to deal with imprecise information. In this 
approach the linear estimations of the individual Kalman filters are improved through a set of the measurement noise covariance matrix $\mathrm{R}$ for each sensor. A priori prediction of the value of $\mathrm{R}$ is done offline using a data sample for each sensor. The fusion of the information is carried out based on the degrees of confidence generated by calculation similarity degree of each sensor data relative to the other sensors. This architecture produced more accurate results than using any one of the individual sensors. In comparison with other methods, the fuzzy similarity-based multi-sensor data fusion of several Kalman filters is able to measure with higher precision in less time and eliminating the uncertainty of measured data. This work can find a wide area of applications specially in the development of radar target tracking, smart structural health monitoring systems, biomedical imaging, and robotics control.

\section{References}

[ 1] Kalman, R. E. 1960. "A New Approach to Linear Filtering and Prediction Problems," Transaction of the ASME—Journal of Basic Engineering, pp. 35-45 (March 1960).

[ 2] Wu, Meiliang, Modeling and on-line system identification for nonlinear structural health monitoring, Ph.D. Thesis , Columbia University, 2006, 122 pages;

[ 3] Chen. G. and Chui. C. K. "A modified adaptive Kalman filler for real-time applications," IEEE Trans. Aerospace and Electronic Systems, 27(1). pp. 149-154. 1991.

[ 4] Grewal MS and. Andrews AP. Kalman Filtering, Theory and Practice Using MATLA0. John Wiley \& Sons, 2001

[ 5] Welch G and Bishop G. An introduction to the Kalman filter. University of North Carolina at Chapel Hill, http://www.cs.unc.edu/-welch

[ 6] Al-Dhaher, A.H.G.; Mackesy, and D. Haptic. "Mufti-sensor data fusion architecture" Audio and Visual Environments and Their Applications. 2004. HAVE 2004. Proceedings. The 3rd International Workshop on 2-3 Oct. 2004 Page(s):159 -163.

[ 7] Young Hwan Lho; Painter, and J.H.; "A fuzzy-tuned adaptive Kalman fitter" Industrial Fuzzy Control and Intelligent Systems. 1993. IFIS '93.. Third International Conference 1 -3 Dec. 1993 Page(s):144 -148 
[ 8] Jetto, L. Longhi, S.; Vitali, D., "Localization of a wheeled mobile robot by sensor data fusion based on a fuzzy logic adapted Kalman filter" Control Engineering Practice, v 7, n 6, June 1999, p 763-71

[ 9] Escamilla-Ambrosio PJ and Mort N. A Hybrid Kalman filter-Fuzzy logic architecture for multisensor data fusion. Proceeding of the 2001 IEEE International symposium on Intelligent Control Mexico City, Mexico 2001,364 369.

[10] Atherton, DP.; Bather, J.A.; Briggs, A.J.; "Data fusion for several Kalman fitters tracking a single target Radar," Sonar and Navigation. IEE Proceedings -Volume 152, Issue 5. October 2005 Page(s): 372 - 376

[11] P.-A. Hebert, M.-H. Massont, T. Denceux, P. Fayet, S. Millemannt and C. Egorofft; "Application of fuzzy preference modelling to the fusion of sensory profile data" 2005 7th International Conference on Information Fusion (FUSION)

[12] Xiong Li; Zongchang Xu; Zhiming Dong; "Fuzzy Similarity-based Data Fusion Algorithm and Its Application to Engine Testing" Granular Computing. 2005 IEEE International Conference on Volume 2, 25-27 July 2005 Page(s):516 - 519 Vol. 2

[13] Mittnik, S. Rachev, S.T.; Samorodnitsky, G., "The distribution of test statistics for outlier detection in heavy-tailed samples," Mathematical and Computer Modelling, v 34, n 9-11, Nov. 2001, p 1171-83.

[14] http://www.itl.nist.gov/div898/handbook/eda/section3/eda35h.htm 